\title{
THE GAMHER RESEARCH PROJECT FOR METRIC DOCUMENTATION OF CULTURAL HERITAGE: CURRENT DEVELOPMENTS
}

\author{
G. Bitelli ${ }^{1}$, C. Balletti ${ }^{2}$, R. Brumana ${ }^{3}$, L. Barazzetti ${ }^{3}$, M. G. D'Urso ${ }^{4}$, F. Rinaudo ${ }^{5}$, G. Tucci ${ }^{6}$ \\ ${ }^{1}$ Department of Civil, Chemical, Environmental and Materials Engineering (DICAM) - University of Bologna, \\ Viale Risorgimento 2, 40136 Bologna, Italy - gabriele.bitelli@unibo.it \\ ${ }^{2}$ Dipartimento di Architettura Costruzione Conservazione, Università Iuav di Venezia, \\ Santa Croce 191, 30135 Venezia, Italy - balletti@iuav.it \\ ${ }^{3}$ Department of Architecture, Built environment and Construction engineering (ABC), Politecnico di Milano, \\ Via Ponzio 3120133 Milano, Italy - (raffaella.brumana, luigi.barazzetti)@ polimi.it \\ ${ }^{4}$ Department of Engineering and Applied Sciences (DISA), University of Bergamo, \\ Viale G. Marconi 5, 24044 Dalmine (BG), Italy - mariagrazia.durso@ unibg.it \\ ${ }^{5}$ Dipartimento di Architettura e Design, Politecnico di Torino. \\ Viale Mattioli 39, 10125 Torino, Italy - fulvio.rinaudo@ polito.it \\ ${ }^{6}$ Geomatics for Environment and Conservation of Cultural Heritage Laboratory, University of Florence, \\ Via Pier Antonio Micheli 8, 50121 Florence, Italy - grazia.tucci@ unifi.it
}

KEY WORDS: Geomatics, Photogrammetry, 3D scanning, CH documentation, Building Information Modeling

\begin{abstract}
:
GAMHer (Geomatics data Acquisition and Management for landscape and built Heritage in a European perspective) is a three-year research project financed under the Italian PRIN 2015 framework (Progetti di Ricerca di Rilevante Interesse Nazionale). The project, started in February 2017 under the University of Bologna coordination, links other 5 Italian research groups mainly involved in Cultural Heritage $(\mathrm{CH})$ documentation and data management: Politecnico di Milano, Politecnico di Torino, IUAV Venice, University of Florence, University of Cassino and Southern Lazio and University of Bergamo.

GAMHer aims at exploiting some research and practical challenges in those disciplines devoted to generate 3D models for objects related to both landscape and built heritage; primary focus is then on digital photogrammetry and 3D scanning. These techniques are more and more applied in different contexts, having as the main subjects monuments, buildings, natural and urban landscapes. Some critical points however remain for their implementation, also facing to the new European directives, strictly connected to the requirement of the Digital Agenda for Europe. A wider use of digital data has to be achieved, in Europe as well as internationally, and therefore an analysis of the current developments is significant, together with a critical assessment of their performances.
\end{abstract}

\section{INTRODUCTION}

When designing the project GAMHer, three main pillars were individuated, and three corresponding Work Packages arranged. The first WP, "Application in maintenance, conservation and enhancement", deals with the very current issues of Historical Building Information Modeling (HBIM) and structural analysis (e.g. FEM), starting from cloud points derived from integrated sources. The complexity of the objects must often be coupled with the simplicity of the input required for other analyses, requiring parametric generation from point clouds or other approaches.

The second WP, "Sharing digital geospatial data on Heritage", addresses some main themes related to the involvement of specialists and non-expert users and to information dissemination. A first theme concerns the possibilities of developing collaborative crowdmapping experiences for Heritage documentation, based on simple protocols for data acquisition - mainly based on Photogrammetry and SfM approaches - and suitable processes of data validation. A second topic deals with the $3 \mathrm{D}$ prototyping by $3 \mathrm{D}$ printers and the metrical characteristics of the obtained models.

The third WP, "Towards best technological practices and quality certification", addresses the quality of the algorithmic approaches and techniques available today, with specific reference to Photogrammetry and Structure from Motion software, in both scientific and commercial products. The spreading use of these systems must be in fact coupled with a significant awareness of their limits and critical aspects. On the other hand, promising studies are towards the direct integration of data coming from photogrammetric surveys and 3D scanning devices.

The results achieved so far in the three Work Packages are promising and will be shown in the next paragraphs.

\section{APPLICATION IN MAINTENANCE, CONSERVATION AND ENHANCEMENT}

One of the aims of the GAMHer project is the investigation of the relationships between Geomatics techniques for data collection/processing and the production of models beyond "pure" geometry. The use of accurate metric information captured with photogrammetric and/or laser scanning methods is crucial for many users in the AEC industry, but metric data must be preliminarily processed to produce usable deliverables which can foster collaboration between different specialists. Such processing must be intended as a digital workflow beyond traditional operations, such as registration of laser scanning point clouds and the creation of dense point clouds from image blocks. Better integration between such products and the type of analyses performed by architects and engineers can be achieved only if the Geomatics specialists can already provide advanced models fulfilling the requirements of the project.

The work in GAMher is currently carried out using an approach on two levels. The first level consists of models at the scale of the building. Experiments were carried out exploiting the 
collaboration with structural engineers for more efficient and rapid production of Finite Element Models. Additional work was carried out using BIM as a platform for the restoration project.

The second level moves the work at a cartographic scale integrating GIS data. Two approaches are currently investigated: 3D City Models as both documentation and change detection instrument, and integrated BIM/GIS models for numerical simulation of road networks.

Finally, the proposal for a novel open source BIM approach is presented. Particular attention is paid to applications in the cultural heritage domain. Such work is illustrated and discussed in the following sections using examples developed by the authors in the framework of the GAMHer project.

\subsection{Some considerations on models at the level of the building}

It is rather obvious that the methods used to produce a model for a specific application are very much depended on the final objective of the application. What is probably less obvious is the quite consolidated "common" strategy for the collection of metric information with photogrammetry and laser scanning technology. Here, the use of dense point clouds has become much less dependent on the application: working at the level of the building requires point clouds for a variety of applications (documentation, productions of drawings, FEA, monitoring, etc.). Nowadays, the procedure for collection and "preliminary processing" of point clouds (e.g., registration, filtering, conversion, decimation, ...) is quite known among the different surveying specialists. This does not mean that anyone can produce a reliable point cloud, but the technological advances in commercial software have reached a significant maturity.

It is the strategy for point cloud transformation into interoperable deliverables that require much research work. For instance, the same set of point clouds must be processed with different approaches to fulfill the requests of structural analysis or a model that represents the building by separating its structural elements. The work described in Bitelli et al. (2018a), concentrates on the need of algorithms able to convert point clouds into Finite Element Models usable by engineers involved in the numerical simulation of structural behavior (Figure 1).

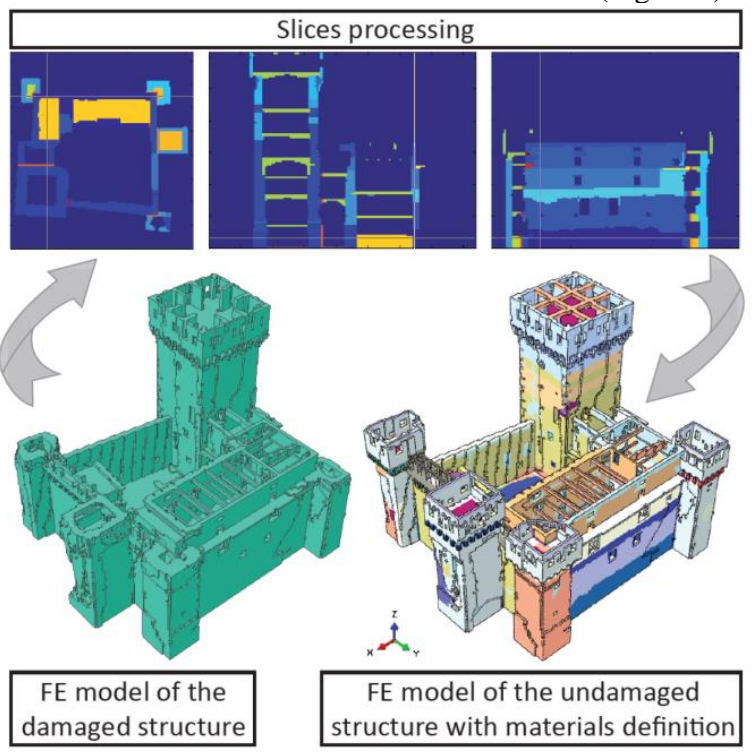

Figure 1. FE models generated from the point cloud: an example of slices processing to obtain the model of the undamaged structure, as well as the material definition.
The transition from point clouds to FEM has required an additional voxel model from pixel-based slices of the original point clouds. Then, the voxel model is converted into a solid hexahedral model. Finally, a nonlinear dynamic analysis was carried out employing the undamaged model.

The second example is mainly connected to the restoration project and decision-making processes among the different actors. The work proposed by Brumana et al. (2018) describes the use of HBIM as a support to the restoration project of the Basilica di Collemaggio. Level of Geometry (LOG) coherent to the Level of Accuracy (LOA) gained by the high-resolution surveys has been adapted to the specificity of the restoration process. The case of a historic monument is proposed through different Grade of Generation (GOG) protocols developed in the object modeling to support the preliminary and definitive design proposal of the conservation plan of the Basilica. Specific Level of Information (LOI) has been introduced to support the preservation process, to document the as-built and the management of the building after the intervention, moving HBIM towards the multi-actor domain.

Further considerations on building models are related to the use of a Bayesian approach that is not based on points cloud but on high-precision topographical measurements in a strategy that has the purpose of accurately estimating the structural displacements expected during the structural life-cycle. Bayesian Networks represent one of the most powerful and effective tools for knowledge acquisition in the observation of physical phenomena affected by randomness and uncertainties. The methodology is the result of several developments concerning the Bayesian statistical theory and permits, by inference, to update the statistics describing physical variables by the observation of experimental evidences. In general, Bayesian Networks have become a very popular and versatile approach in problem solving strategies because of their capability of enhancing the status of knowledge of a physical problem domain and to characterize expected outcomes (D'Urso et al., 2017).

\subsection{Considerations on models at cartographic scale}

Existing cartographic information coupled with point clouds acquired by photogrammetry or LiDAR opens new opportunities for applications requiring 3D models at the level of the built environment. The work carried out in GAMHer focused on two approaches: (i) 3D City Model and (ii) BIM for infrastructures. Both make use of existing information in raster or vector formats (e.g., orthophotos, digital terrain models, shapefiles, ...) used with classified LiDAR or dense photogrammetric point clouds. Although the same input data are exploited by both methods, the idea for the production of the final model is rather different.

Bitelli et al. (2018a) used a point cloud to generate a LOD2 City Model (Figure 2) for the coastal area of the city of Riccione (Italy). The low density of the LiDAR point cloud (not originally acquired to produce a city model) prevented the production of a model with a superior level of detail. Starting from the classified point clouds combined with the DTM and urban numerical cartography, a 3D City Model was generated. The model can reveal the actual elevation of each building in an interactive three-dimensional environment. The proposed workflow was completely automated, and it proved to be a fast and effective processing method to rapidly deliver results. It is also easily replicable in other cases of study providing a new use of already surveyed datasets. 


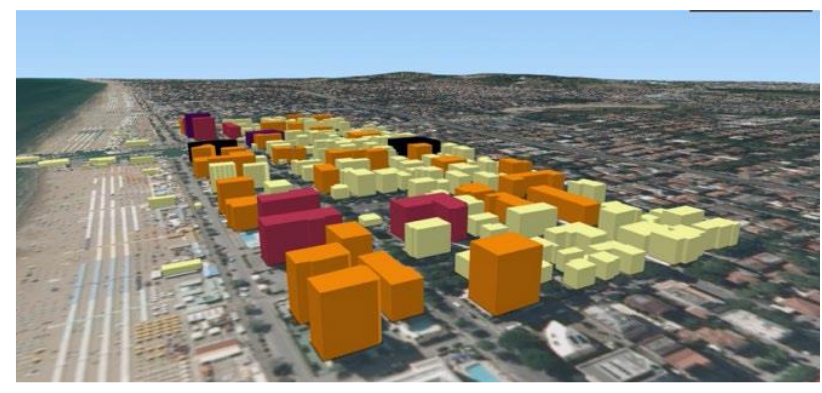

Figure 2. 3D City Model graphical representation with LoD1 buildings classified according to their new volume attribute.

The work proposed in Barazzetti (2018) was done to test the interoperability between GIS and BIM systems for projects at the level of infrastructures. Geographic Information System (GIS) and Building Information Modelling (BIM) can be very useful when the work requires different scales. For instance, BIM sw are mainly designed for the scale of the building, and GIS for the district, city, regional, national and international scales. On the other hand, such distinction is not always valid since many examples with GIS sw were carried out also for small objects. Another distinction could be based not only on the type of data, but also on the type of operations. GIS engines have advanced functions for geospatial analysis, whereas BIM sw have advanced parametric modeling tools. A common feature of both technologies is the database connected to the graphic representation of specific objects.

After reviewing common aspects and differences between BIM and GIS, the work ended with the simulation of traffic conditions with different configurations of the bridge Azzone Visconti in Lecco. In particular, the aim was to investigate the usability of an integrated BIM/GIS model for a traffic simulation in the area around the bridge. Results highlighted that traditional BIM (at the level of construction) must be replaced with another integrated BIM-GIS solution (Figure 3) more suitable for analysis based on geospatial data.

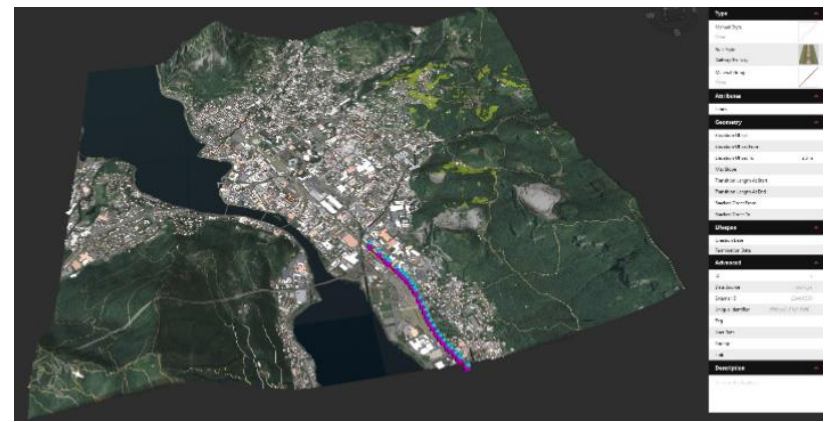

Figure 3. Integrated BIM/GIS model for the city of Lecco.

\subsection{Towards Open Source HBIM}

Diara and Rinaudo (2018) recently proposed a novel approach for an Open Source Historic BIM (HBIM). The adoption of an open source approach is a milestone in data preservation and fruition, while closed formats are prone to continuous upgrades by the owners and can disappear and lose their portability on other platforms.

Now, the world of BIM is dominated by commercial applications (e.g., Autodesk Revit, ArchiCAD, Tekla BIMsight, etc.) and the use of open source and free services could be considered quite challenging. However, a suitable solution to guarantee the best and complete data usability and accessibility could be the use of new open source protocols, both for BIM software and BIM file formats. This choice would allow adapting software and DBMS to the Cultural Heritage needs and not the opposite, thus avoiding methodological stretches. Also, in the Cultural Heritage field (like archaeology), many analysis and researches carried out using FOSS instruments and software have often proved to be quite reliable, allowing to obtain more complete results than other research works.

The aim of an Open Source BIM proposal is the future creation of a dynamic and open system, maybe based on FOSS solutions that could be adopted without commercial restrictions.

\section{SHARING DIGITAL GEOSPATIAL DATA}

\subsection{Spreading data acquisition and digitalization processes}

The constant technological evolutions in Geomatics techniques has been leading to a democratization process associated also to a progressively increase in automatized procedures, thus becoming more and more accessible to a mass audience (Fassi et al., 2017; Tucci et al., 2017).

In this context, photogrammetry is the most user-friendly technique in terms of both device availability and ease of use: the process of obtaining 3D models by simply acquiring pictures with common cameras and processing them by automatic user-friendly software seems a very attractive tool for both professional and amateur users. Architects, engineers, archaeologists, geologists, agronomists, computer-vision artists are very attracted to and interested in the outputs of dense point clouds or surface models, without a specific education or training to understand the rigorous processes behind the automatisms. This democratization process is like an inexorable flow that cannot be opposed, both thanks to the rapid evolution of mobile devices, equipped with increasingly high-resolution sensors, and to the work made in past years to develop strong and efficient algorithms for automatic data processing (Tucci, 2018a; Tucci 2018b).

Furthermore, photogrammetry is characterized by low-cost acquisition devices (almost any kind of cameras and operative costs, suitable for an extensive and flexible utilization, compared to more expensive and bulkier geomatics tools (e.g. laser scanner). This progressively opening to a wider audience, not only specialists, however entails the risk of lack of quality and awareness of the metric control over the resulting outputs.

This panorama requires to adopt a series of precautions, by setting up a control frame system which should help guarantee a minimum level of quality, where divulgation and education play key roles. The accessibility to geomatics technology to nonexpert users could not be considered only negatively. As stated by the Cultural Heritage Imaging project mission (http://culturalheritageimaging.org/About_Us/Mission/), a crowdsourced approach can help in the documentation and preservation processes of Cultural Heritage (McCarthy, 2014), by directly involving people passionate about saving their own at-risk heritage. This vision generates a virtuous circle under the social aspect because it empowers people's awareness of their culture by triggering collective consciousness, identification and engagement feelings (Chiesi et al., 2015, Latellier, 2015). Furthermore, the aim is also to let people know the existence of such techniques, to trigger the potential interest in geomatic technologies that they even don't know to habitually use (as GPS and so on).

The social aspect, however, should be supervised by experts and specialists, who provide tools, technology, and training by the diffusion of best practices, shared standards and suitable guidelines to a broad audience, thus keeping a sort of control over the technological transfer to non-experts. 
The final application is the organization of events, as workshops, tutorials or crowdsourced activities, where the basics of the technique to assure a minimum level of quality are illustrated, followed by practical activities on the field for data acquisition of selected themes, useful for digitization processes of Cultural Heritage (Figure 4). The processing phase of the acquired data can be carried out directly by experts but showing the final outputs on suitable platforms, as websites or mobile app, to give the participants an idea of what they have produced starting from "simple" pictures.
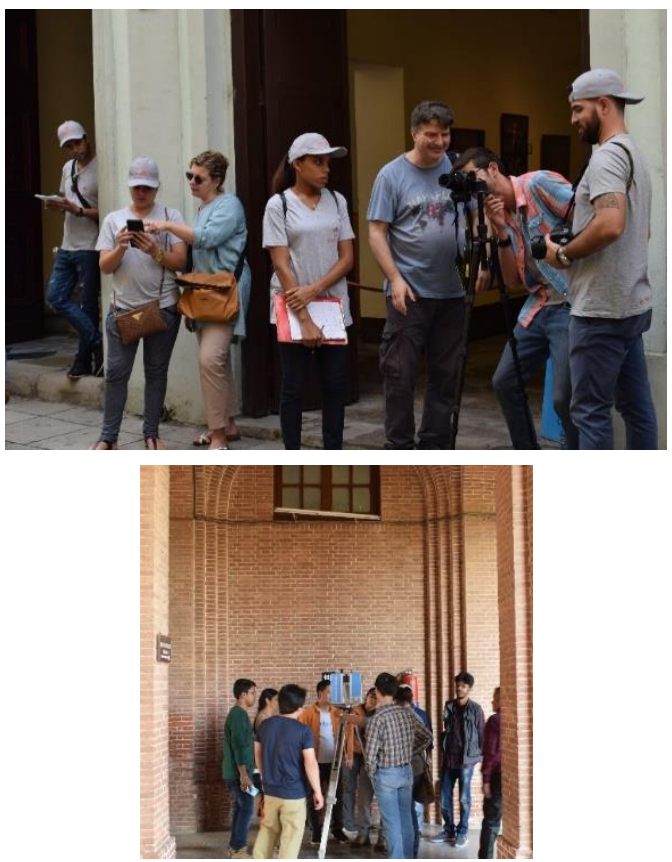

Figure 4. On the field activities during workshops and tutorials in Havana (Cuba) and Dehradun (India) to give the participants a basic introduction on integrated surveys with geomatics techniques followed by practice activities.

Moreover, the creation of multimedia databases, their insertion into a GIS platform, and the realization of 3D digital models of sites and architectural structures, are the substrate on which additional applications can be developed. An example is represented by the GIS platform presented in (D'Urso et al., 2018) that allows one to consult a huge metric and typological database relative to archaeological sites and significant monuments of the Aquino "Vallone", in the Southern Lazio, mainly related to the early medieval period, an area never surveyed in the past. An even closer collaboration among Universities, research institutes, Superintendents for Cultural Heritage and local authorities can lead to further developments of the ambitious Open Project on the Roman and Medieval Aquino, which since 2015 has fostered progresses in the field of protection, conservation and diffusion of the town's history and archaeological heritage. Laser-scanning, photogrammetry and computer vision are very useful tools for archaeologists, although a suitable integration of the representation of space and archaeological time has not yet found a methodological standard of reference.

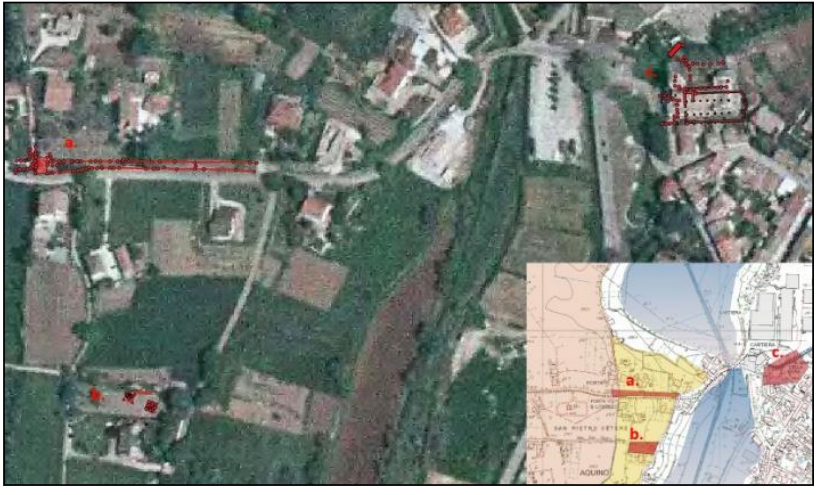

Figure 5. GPS survey, screenshot of the GIS, basemap ortophoto Regione Lazio 1:2000: a) Porta San Lorenzo and Via Latina; b) Excavations of Madonna degli Angeli Church; c) Santa Maria della Libera Church and Marco Antonio's Arch (D'Urso et al., 2017).

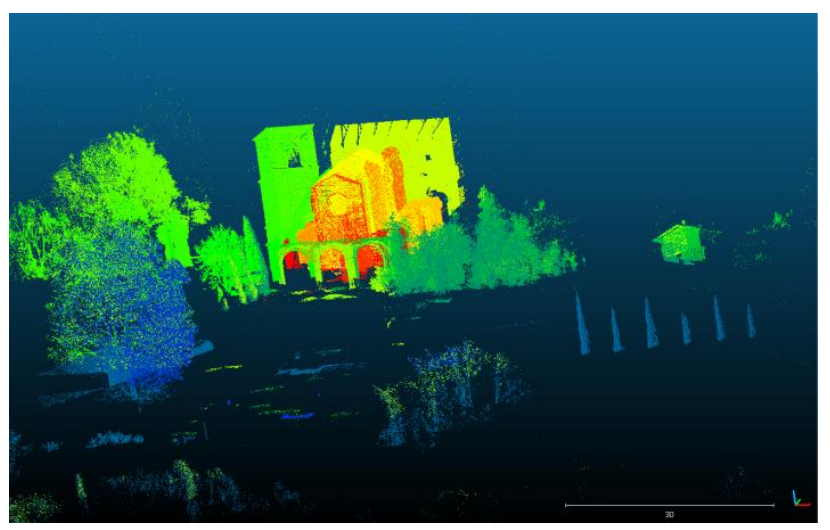

Figure 6. Points cloud in the area of S. Maria della Libera Church (D'Urso et al., 2018)

\subsection{D Printing and quality evaluation}

$3 \mathrm{D}$ printing is one of the latest developments within the range of technologies applied to the $\mathrm{CH}$ context. However, it is only one step in the extensive process of data acquisition and management: the final moment of the workflow, from recording the shape of the object to its physical reproduction. Because it is part of this process, the technologies of solid printing are closely linked to those for metric data acquisition (point cloud production) and digital data management (virtual modelling).

As a result, the literature on digital modelling applications tends to be intermingled with that on solid printing. In fact, the more general term "3D technologies" now covers a wide range of tools, methods and applications of modelling and printing.

The use of printing processes based on metric surveying enables production of objects that replicate the true shapes. The "point cloud", meaning the digital point model obtained by photogrammetry or laser scanning, can be saved and archived, providing a record of the true state of an object in a given spacetime context, and these models can then also be modified or replicated as desired. Researchers often use these threedimensional digital models and printed replicas for purposes of data collection and analysis, thereby avoiding physical contact with sensitive objects and exhibits (Ioannides et al., 2010; Ioannides and Quak, 2014; Francolini et al., 2018).

As noted, the most rapid and extensive diffusion of $3 \mathrm{D}$ technologies, within the overall area of $\mathrm{CH}$, has been in museums. In this sector, the concept of "usability" of $\mathrm{CH}$ has always been manifest: a reality that relates well the potentials of 
3D technologies for the attraction and engagement of a very wide public, including even those who would not be regular visitors. The most avant-garde institutions now recognise modern technologies as an opportunity to add new "reading" tools to their traditional visitor. The aim is to move the traditionally conceived museum exhibit towards a multi-level and multi-sensory experience (Petrelli et al., 2013; Wilson et al., 2017). Touch and manipulation of objects, whether physical or virtual, offers the advantages of creating a stronger connection than mere sight, between the individual and the object. In this context, 3D technologies provide a privileged vehicle for engaging of users with material culture. Haptic interfaces and printed replicas can enable both tactile and kinaesthetic feedback (D'Agnano et al. 2015), and simulate the physical properties of original materials: the user can go beyond the simple appreciation of shape and colour, to now sense the weight, structure, texture and friction of the object.

Given its remarkable development in other fields, interest in solid printing has also extended to the sphere of geomatics, meaning the gathering, processing and delivering spatially referenced geographic information: so much that 3D printing, similarly to paper or digital products, is considered among potential outputs when planning a survey. However, the path from the surveyed model to its physical representation implies a series of steps that result in simplifications, and so the loss of conformity between the original object and its reproduction.

The purpose of the case studies is to analyse the criticalities in the path from the surveying to the physical representation of the object, concentrating on potential problems in data acquisition and processing, and then in the metric precision of the resulting 3D printing (e.g. Figure 7). Some applications, drawn from a series of cases (Adami et al, 2015; Balletti et al., 2016; Balletti al., 2017; Ballarin et al., 2018) dealt with over the course of the past year, were selected on the basis of their ability to illustrate the main problems in the technological path. The examples deal with the acquisition of data from two objects of different shapes and sizes, using different methodologies and tools, which then lead to different methods of data processing.
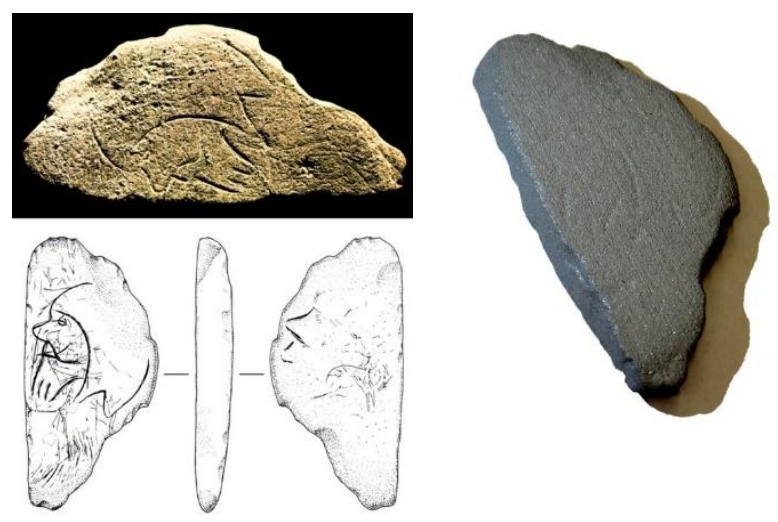

Figure 7. Image and drawings of the limestone slab of the "Uomo barbuto di Vado all'Arancio" and its replica in Alumide.

\section{BEST PRACTICES AND QUALITY CERTIFICATION}

In the traditional approach to metric survey, adopted up to 20 years ago, the surveyors selected the points needed to build up the 3D models during the acquisition, therefore the precision and accuracy of the metric data were transferred to the $3 \mathrm{D}$ model without any degradation of those properties.

The today trend to build up 3D models of movable objects, complex architectural assets and natural landscapes comes from the availability of automatic tools able to survey sparse and dense 3D point clouds (e.g. digital photogrammetry, LiDAR, integrated systems, etc.).

Those 3D point clouds represent just the starting point of the survey which main aim is to define 3D models with certified precision and accuracies and allowing to build up different kind of 3D models or traditional 2D drawings.

The introduction of automatic tools able to manage big amounts of data gave not only positive advances in metric survey approach. Due to the fact that software is developed by people not experts in metric survey and the continuous look for almost autonomous procedures, all the step by step check of precision disappeared giving the illusion to unskilled people to be able to manage a metric survey just by clicking some buttons in a predefined order. The subsequent modelling is performed by considering the point clouds as "perfect" data instead as physical measurements as they really are.

If we consider also that sparse point clouds could be modeled by using automatic tools based on Delaunay algorithms the risk to have metric surveys without "intelligence" is clear.

GAMHer project aimed to show that correct metric surveys needs an intelligence behind every step of the procedure: from the acquisition of the point clouds, to the merging of point clouds with different precision and resolution, to the modeling of complex objects by considering the final aim of the survey and a correct understanding of the geometric complexity of the surveyed asset. All these three phases require a complete knowledge of the measurement problems and of the final goals of the survey which define the deliverables to be produced and the needed level of accuracy and resolution to be reached.

\subsection{Precision assessment}

Image based acquisition techniques would allow the assessment of the reached precisions at the end of the survey while distance based techniques do not allow it. In those last cases just a predictable precision is used to characterize the precision of the obtained 3D point cloud.

Today software, both on photogrammetric and LiDAR primary data management, do not take care too much about the estimation of the achieved precisions and usually they neglect to show precision indicators during the different steps to allow a possible intervention during the data processing itself.

Image based software usually do not allow to analyze the achieved precision in the different steps of a photogrammetric process: matching results, camera calibration, relative and absolute orientations. In the last years some of them show main statistics about re-projection discrepancies after relative orientation of the obtained unscaled 3D point clouds and the discrepancies on Ground Control Points (GCPs) after absolute orientation: this fact could be accepted as a positive development of automatic photogrammetric tools and platforms (Calantropio et al., 2018).

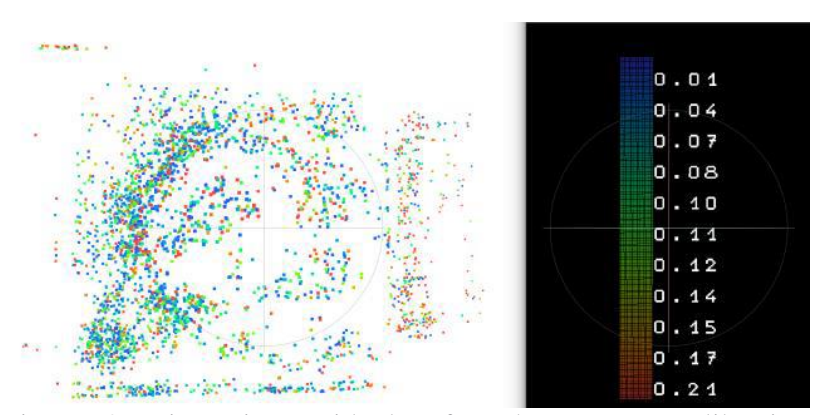

Figure 8. Tie point residuals after the camera calibration (Calantropio et al. 2018) 
Similar approaches are now possible for 3D sparse point cloud extraction from video frames as natural development of the videogrammetry solutions adopted in the past.

Distance based software in some cases allow to analyze the discrepancies of targeted points used for the point cloud registration also if automatic Iterative Closest Point (ICP) based procedures are used (Calantropio et al, 2018).

Surveyors know that only a precision assessment is not enough to certify the quality of a metric survey: systematic errors could affect in some ways the achieved results in all the measurement processes (Condorelli et al, 2018).

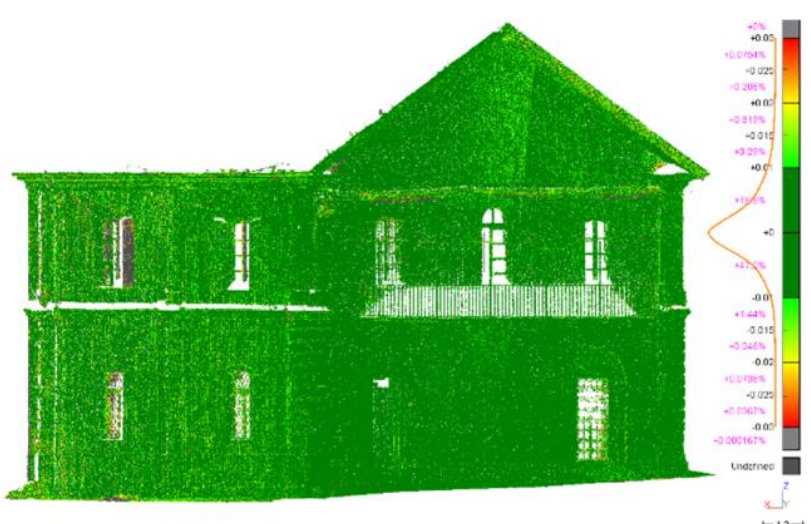

Figure 9. Cloud-to-Cloud comparison (Calantropio et al., 2018)

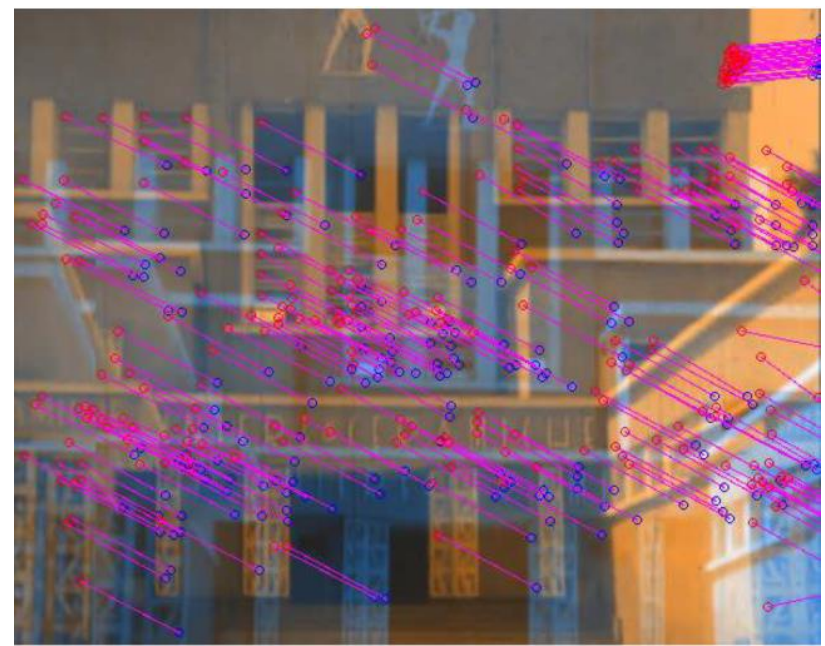

Figure 10. Tie Point selection between two adjacent video frames (Condorelli et al., 2018)

Therefore, an accuracy assessment is always needed and it requires the knowledge of some basic geometric quantities (e.g. coordinates of Check Points or distances).

Accuracy tests could be performed on the acquired point clouds if considered CPs or distances are correctly materialized. Another possible check of the point cloud accuracy could be set up by comparing the new point cloud with an existing point cloud with a precision that must be greater than the one expected form the new one.

The metric survey ends when the final 3D model has been set up therefore, an additional accuracy test must be provided on the 3D model. Actually the vertexes of the 3D model do not coincide generally with a point of the used point clouds but they are generated by interpolating the point clouds. It is well known that the interpolation of measurement generates a less precise measurement, therefore a final accuracy test is needed at the end of the whole metric survey process.

\subsection{Different precision and resolution in point clouds merging}

Cultural Heritage assets are usually complex in shape and different environmental conditions push the surveyors to use many point cloud acquisition systems characterized by different precision and resolution (e.g. automatic digital photogrammetry, LiDAR, SLAM based systems, etc.).

GAMHer units developed some complete 3D metric surveys in different cases, by considering that general rules cannot be stated for all the possible kind of Cultural Heritage assets (Brumana et al., 2017; Tucci et al., 2017).

HBIM protocols (Brumana 2018a) have been performed in order to manage the complexity of the architectural heritage objects and to match the different requirements coming from the different actors (BIM-FEA, BIM-EE, BIM-LLCCM)

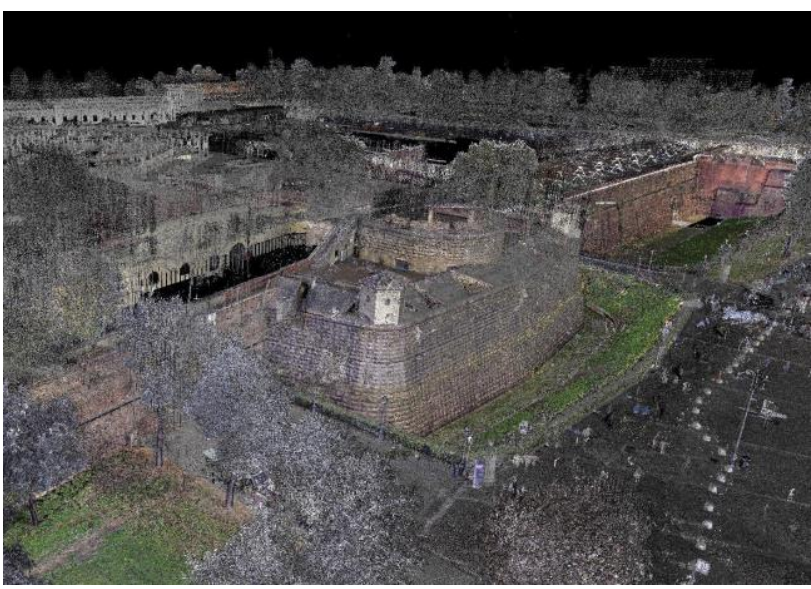

Figure 11. Point cloud generated by different sensors (Tucci et al., 2017)

All the achieved experiences show and demonstrate the need of point clouds with a precision and resolution higher than the one expected for the final 3D model to avoid the loss of precision due to the modeling steps (segmentation and surface interpolation). When the used point clouds have different level of precision the less precise could be adapted (by scaling and translating them) to the most precise ones.

NURBS based Grade of Generations (GOGs) have been defined (Banfi, 2017) to take in account the richness and complexity of the object elements, as in the case of damaged walls (Figure 12) of vaulted systems (Brumana et al., 2018b).

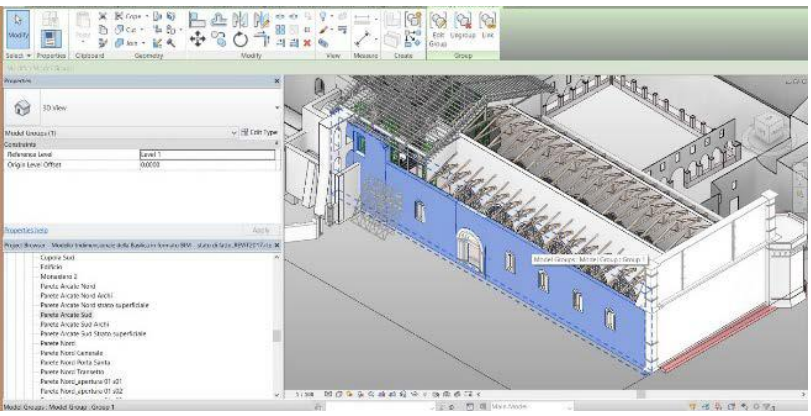

Figure 12. NURBS based BIM modeling (Brumana et al, 2018a) 
In case of different resolution, the efforts to be used during the modelling phase is greater and require a complete knowledge of the surveyed object and a clear understanding of the final goals of the survey.

\section{CONCLUSIONS}

GAMHer project tries to interface with some of the most challenging themes in the field of metric data acquisition, processing and modelling for Cultural Heritage. The addressed open questions are at different levels: the intrinsic quality of new procedures for point cloud acquisition, the modelling phase towards HBIM or 3D GIS establishment, the problems related to physical 3D models and their generation, and, finally, the involvement of non-experts in collaborative photogrammetric surveying. From the results so far achieved, interesting results come out that should lead to a higher level of quality in the surveying and representation of Heritage.

\section{ACKNOWLEDGEMENTS}

This work has been carried out under the GAMHer project: Geomatics Data Acquisition and Management for Landscape and Built Heritage in a European Perspective, PRIN: Progetti di Ricerca di Rilevante Interesse Nazionale - Bando 2015, Prot. 2015HJLS7E. GAMHer Website: https://site.unibo.it/gamher/en

\section{REFERENCES}

Adami, A., Balletti, C., Fassi, F., Fregonese, L., Guerra, F., Taffurelli, L., Vernier, P., 2015. The bust of Francesco II Gonzaga: from digital documentation to 3D printing. ISPRS Ann. Photogramm. Remote Sens. Spatial Inf. Sci.; II-5/W3, pp. $9-15$.

Ballarin, M., Balletti, C., Vernier, P., 2018. Replicas In Cultural Heritage: 3D Printing and the Museum Experience. Int. Arch. Photogramm. Remote Sens. Spatial Inf. Sci., XLII-2, pp. 55-62.

Balletti, C., Ballarin, M., Guerra, F., 2017. 3D printing: state of the art, considerations and future perspectives. Journal of Cultural Heritage, 26, pp. 172-182.

Balletti, C., D'Agnano, F., Guerra, F., Vernier, P., 2016. From point cloud to digital fabrication: a tangible reconstruction of $\mathrm{Ca}$ ' Venier dei Leoni, the Guggenheim Museum in Venice. ISPRS Ann. Photogramm. Remote Sens. Spatial Inf. Sci., III-5, pp. 43-49.

Banfi, F., 2017. BIM orientation: grades of generation and information for different type of analysis and management process. In: The International Archives of the Photogrammetry, Remote Sensing and Spatial Information Sciences, 42(2/W5), pp. 57-64.

Barazzetti, L., Banfi, F., 2017. BIM And GIS: When Parametric Modeling Meets Geospatial Data. ISPRS Ann. Photogramm. Remote Sens. Spatial Inf. Sci., IV-5/W1, pp. 1-8.

Bitelli, G., Castellazzi, G., D’Altri, A.M., De Miranda, S., Lambertini, A., Selvaggi, I., 2018a. On the generation of numerical models from point clouds for the analysis of damaged Cultural Heritage. IOP Conf. Series: Materials Science and Engineering, 364, 012083.

Bitelli, G., Girelli, V.A., Lambertini, A., 2018b. Integrated use of Remote Sensed Data for the Generation of Accurate and Up- to-date 3D City Models. Int. Arch. Photogramm. Remote Sens. Spatial Inf. Sci., Volume XLII-2, pp. 97-102.

Brumana, R., Della Torre, S., Oreni, D., Previtali, M., Cantini, L., Barazzetti, L., Franchi, A., and Banfi, F., 2017. HBIM Challenge Among The Paradigm Of Complexity, Tools And Preservation: The Basilica Di Collemaggio 8 Years After The Earthquake (L'Aquila). Int. Arch. Photogramm. Remote Sens. Spatial Inf. Sci., XLII-2/W5, pp. 97-104.

Brumana, R., Della Torre, S., Previtali, M., Barazzetti, L., Cantini, L., Oreni, D., Banfi, F., 2018a. Generative HBIM modelling to embody complexity (LOD, LOG, LOA, LOI): surveying, preservation, site intervention-the Basilica di Collemaggio (L'Aquila). Applied Geomatics, Vol. 10, 4, pp. 545-567.

Brumana, R., Condoleo, P., Grimoldi, A., Banfi, F., Landi, A.G., Previtali, M., 2018b. HR LOD based HBIM to detect influences on geometry and shape by stereotomic construction techniques of brick vaults. In: Applied Geomatics 10, pp. 529543. DOI 10.1007/s12518-018-0209-3, Special Issue GEORES2017. Geomatics and Restoration: Conservation of Cultural Heritage in the Digital Era ,Springer.

Calantropio, A., Deseilligny, M.P., Rinaudo, F., and Rupnik, E., 2018: Evaluation of photogrammetric block orientation using quality descriptors from statistically filtered tie points. Int. Arch. Photogramm. Remote Sens. Spatial Inf. Sci., XLII-2, pp. 185-191.

Calantropio, A., Chiabrando, F., Rinaudo, F., Teppati Losè, L., 2018: Use and evaluation of a short range small quadcopter and a portable imaging laser for built heritage 3D documentation. Int. Arch. Photogramm. Remote Sens. Spatial Inf. Sci., XLII-171, pp. 71-78.

Chiesi, L., Costa, P., 2015. One strategy, many purposes. A classification for cultural mapping projects, Cultural Mapping as Cultural Inquiry, Routledge, London, pp. 69-85.

Condorelli, F., Rinaudo, F., 2018: Cultural Heritage reconstruction from historical photographs and videos, Int. Arch. Photogramm. Remote Sens. Spatial Inf. Sci., XLII-2, pp. 259-265.

D’Agnano, F., Balletti, C., Guerra, F., Vernier, P., 2015. TOOTEKO: a case study of augmented reality for an accessibile cultural heritage. Digitization, 3D printing and sensors for audio-tactile experience. Int. Arch. Photogramm. Remote Sens. Spatial Inf. Sci.; XL-5/W4, 2015, pp. 207-213.

Diara, F., Rinaudo, F., 2018. Open Source HBIM for Cultural Heritage: A Project Proposal, Int. Arch. Photogramm. Remote Sens. Spatial Inf. Sci., XLII-2, 303-309.

D'Urso, M.G., Gargiulo, A., Sessa, S., 2017. A Bayesian approach for controlling structural displacements. XXIV International Conference "Mathematical and Computer Simulations in Mechanics of Solids and Structures", Procedia Structural Integrity 6, (2017), pp. 69-76.

D’Urso, M.G., Corsi, E., Corsi, C., 2018. Mapping of archaeological evidences and 3D models for the historical reconstruction of archaeological sites. Proceedings IEEE International Workshop on Metrology for Archaeology and 
Cultural Heritage (MetroArchaeo), IEEE Catalog Number: CFP18O73-USB; ISBN: 978-1-5386-5275-6, pp: 432-437.

D'Urso, M.G., Marino, C.L., Rotondi, A., 2018. Close-range Imagery for Reconstructing Archaeological Findings. Proceedings IEEE International Workshop on Metrology for Archaeology and Cultural Heritage (MetroArchaeo), IEEE Catalog Number: CFP18O73-USB; ISBN: 978-1-5386-5275-6, pp.188-193.

Fassi, F., Campanella, C., 2017. From daguerreotypes to digital automatic photogrammetry. Applications and limits for the built heritage project. In: Int. Arch. Photogramm. Remote Sens. Spatial Inf. Sci., Vol. XLII-5/W1, pp. 313-319.

Francolini, C., Marchesi, G., Bitelli, G., 2018. High-resolution 3D survey and visualization of Mesopotamian artefacts bearing cuneiform inscriptions. Proceedings IEEE International Workshop on Metrology for Archaeology and Cultural Heritage (MetroArchaeo), ISBN: 978-1-5386-5275-6, 178-182.

Ioannides, M., Fellner, D., Georgopoulos, A., Hadjimitsis, D.G., 2010. Digital Heritage. In Proc. of the Third International Conference, EuroMed, Lemessos, Cyprus, Nov. 8-13.

Ioannides, M., Quak, E., 2014. 3D Research Challenges in Cultural Heritage. A Roadmap in Digital Heritage Preservation. Springer-Verlag Berlin Heidelberg.

Letellier, R., 2015. Recording, documentation and information management for the conservation of heritage places. Routledge, London/New York.

McCarthy, J., 2014. Multi-image photogrammetry as a practical tool for cultural heritage survey and community engagement. J. of Archaeological Science, 43, pp. 175-185.

Petrelli, D., Ciolfi, L., Van Dick, D., Horneker, E., Not, E., Schmidt, A., 2013, Integrating material and digital: a new way for cultural heritage. Interactions, 20, pp. 58-63.

Tucci, G., Bonora, V., Conti, A., Fiorini, L., 2017. High-quality 3D models and their use in a cultural heritage conservation project. In: Int. Arch. Photogramm. Remote Sens. Spatial Inf. Sci., Vol. XLII-2/W5, pp. 687-693.

Tucci, G., Conti, A., Fiorini, L., Mei, F., and Parisi, E. I., 2018a. Digital photogrammetry as a resource for Cuba Cultural Heritage: educational experiences and community angagement within the INNOVA CUBA project, Int. Arch. Photogramm. Remote Sens. Spatial Inf. Sci., XLII-5, pp. 37-44.

Tucci, G., Conti, A., Fiorini, L., Panighini, F., and Parisi, E. I., 2018b. Education and training resources on digital photogrammetry, Int. Arch. Photogramm. Remote Sens. Spatial Inf. Sci., XLII-5, 45-50.

Wilson, P.F., Stott J., Warnett, J.M., Attridge, A., Smith, M.P., Williams, M.A., 2017. Evaluation of touchable 3d-printed replicas in museums. Curator Museum Journal, 60, 4, pp. 445465. 